\title{
Visualization of the unburned gas flow field ahead of an accelerating flame in an obstructed square channel
}

\author{
Craig T. Johansen* and Gaby Ciccarelli \\ Mechanical and Materials Engineering, Queen’s University, Kingston, ON K7L 3N6, Canada \\ *Corresponding Author: $\quad$ Craig T. Johansen \\ Now at University of Calgary \\ Email: johansen@ucalgary.ca
}

\begin{abstract}
The effect of blockage ratio on the early phase of the flame acceleration process was investigated in an obstructed square cross-section channel. Flame acceleration was promoted by an array of top and bottom-surface mounted obstacles that were distributed along the entire channel length at an equal spacing corresponding to one channel height. It was determined that flame acceleration is more pronounced for higher blockage obstacles during the initial stage of flame acceleration up to a flame velocity below the speed of sound of the reactants. The progression of the flame shape and flame area was determined by constructing a series of three dimensional flame surface models using synchronized orthogonal schlieren images. A novel schlieren based photographic technique was used to visualize the unburned gas flow field ahead of the flame front. A small amount of helium gas is injected into the channel before ignition, and the evolution of the helium diluted unburned gas pocket is tracked simultaneously with the flame front. Using this technique the formation of a vortex downstream of each obstacle was observed. The size of the vortex increases with time until it reaches the channel wall and completely spans the distance between adjacent obstacles. A shear layer develops separating the core flow from the recirculation zone between the obstacles. The evolution of oscillations in centerline flame velocity is discussed in the context of the development of these flow structures in the unburned gas.
\end{abstract}

\section{Keywords}

Flame Acceleration; Schlieren Photography; Obstacle Flow; Explosions

\section{Introduction}

Flame propagation in obstructed channels has been studied for many years [1], primarily in connection with explosion safety. The interaction of the flame and the unburned gas flow field generated ahead of the flame provides an efficient feed-back loop that can lead to flame acceleration up to a velocity relative to a fixed observer on the order of the speed of sound of the combustion products, i.e., roughly $1000 \mathrm{~m} / \mathrm{s}$. Initially the geometric increase in flame area caused by the large-scale flow structures produced by the obstacles is responsible for flame acceleration. As flame acceleration proceeds and the unburned gas flow becomes turbulent, the transport of mass and momentum into the flame is augmented and the resulting increase in the burning rate further enhances flame acceleration. As a result of these two effects, strong flame acceleration can lead to the production of a precursor shock wave with overpressures on the order of the adiabatic constant volume explosion pressure. In this last stage shock flame 
interactions can lead to severe flame distortion which further supports flame acceleration and in extreme cases, causes transition to detonation. The process of detonation initiation occurring at the culmination of flame acceleration is referred to as deflagration-to-detonation transition (DDT). Comprehensive reviews of flame acceleration and DDT can be found in [2-4].

The flame acceleration process is governed by several parameters, including mixture reactivity and the ratio of the obstacle blockage area and the channel cross-section area, i.e., BR [5]. Vesser et al. [6] performed experiments in a circular tube quipped with different BR orifice plates and performed corresponding three-dimensional gasdynamic simulations. The calculations did not include turbulence and assumed a constant burning velocity equal to ten times the laminar burning velocity. Based on a comparison of the experimental and numerical results, they showed that a "choked" flame can be generated by flame area enhancement resulting from the interaction of the unburned gas flow ahead of the flame with the obstacles. A choked flame is a flame that propagates at a velocity limited to the speed of sound of the combustion products. It can be shown via a one-dimensional, double-discontinuity (flameshock) analysis that a critical flame velocity corresponds to the condition where the flame velocity equals the speed of sound in the products [7]. This flame velocity is important because for values less than this the combustion products remain at rest as there is communication between the flame and the closed end where the gas must be stationary. For flame velocities above this critical value an expansion fan must be present to drop the particle velocity to zero at the closed end. Vesser et al. [6] proposed a simple analytical model that describes the evolution of the flame surface area and predicts the choked flame run-up distance.

In most studies flame propagation velocity down the length of the channel is deduced from the flame time-of-arrival measured by ionization and photodiode probes. Piezoelectric pressure transducers are used to record the pressure transient at different axial locations in the channel. This pressure data can be used to track the development of the shock wave ahead of the flame and the onset of detonation. More recently, high-speed schlieren video has been used to capture the complex turbulent flame shape during flame acceleration [8]. As discussed above the flame shape is dictated predominately by the unburned gas flow-field ahead of the flame, which is dominated by turbulent flow structures such as large eddies and shear layers produced downstream of obstacles [9]. To get a better understanding of the flame propagation mechanism it is imperative to characterize the flow field ahead of an accelerating flame for different channel geometries.

In this study, a novel schlieren based photographic technique, where helium gas is injected ahead of the flame, is used as a tracer to provide a two-dimensional map of the flow field structure ahead of the flame. With knowledge of the flow field structure ahead of the flame insight can be obtained concerning the evolution of the flame surface resulting from the interaction of the flame with this flow field. The objective of the study is to investigate the early stage of the flame acceleration process, i.e., up to flame velocities on the order of the speed of sound of the reactants, and not the DDT phenomenon. The channel height and width are smaller than the detonation cell size for the mixture used and thus detonation initiation is not expected to occur.

\section{Experimental setup}

Experiments were performed in a modular aluminum 6061-T6 combustion channel shown in Fig. 1. The $2.44 \mathrm{~m}$ long channel is comprised of three non-optical modules and one 
optical module, each with a square cross-section of $7.6 \mathrm{~cm} \times 7.6 \mathrm{~cm}$. Each non-optical module is equipped with two instrumentation ports spaced $30.5 \mathrm{~cm}$ apart and $15.2 \mathrm{~cm}$ from the end flanges on both the top and bottom surfaces. The instrumentation configuration for all of the flame acceleration tests involved four piezoelectric pressure sensors mounted in instrumentation ports on the top surface. Ionization probes were mounted in each available instrumentation port on the bottom channel surface and protruded $1.5 \mathrm{~cm}$ into the test section.

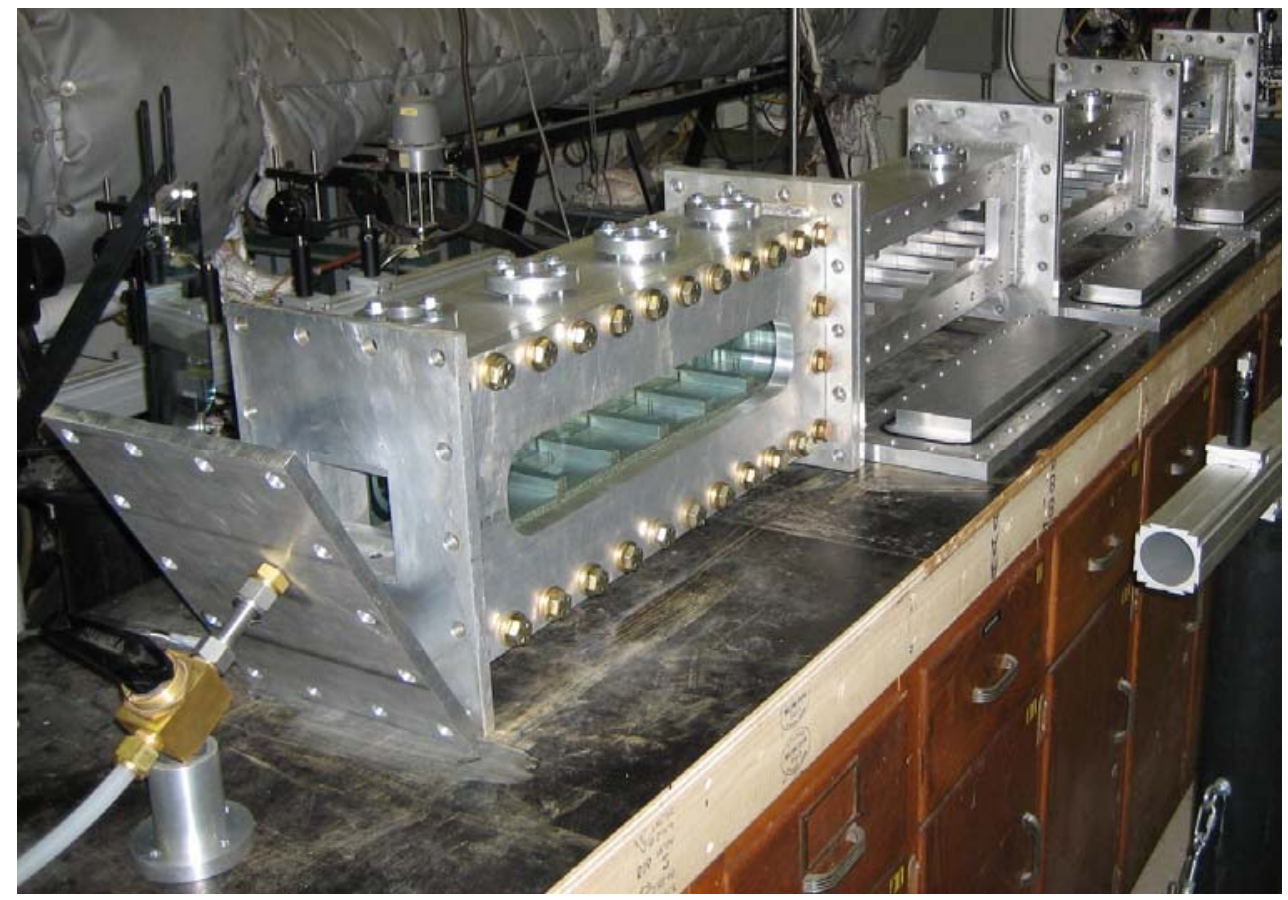

Figure 1: Experimental apparatus

A schematic of the optical module shown in Fig. 2 indicates the position of eight instrumentation ports spaced $15.2 \mathrm{~cm}$ apart and $7.6 \mathrm{~cm}$ from each end flange. Two, $1.9 \mathrm{~cm}$ thick glass windows are integrated into the channel front and back sides to facilitate $7.6 \mathrm{~cm} \times 44.5 \mathrm{~cm}$ of optical access for the visualization of the flame acceleration process.

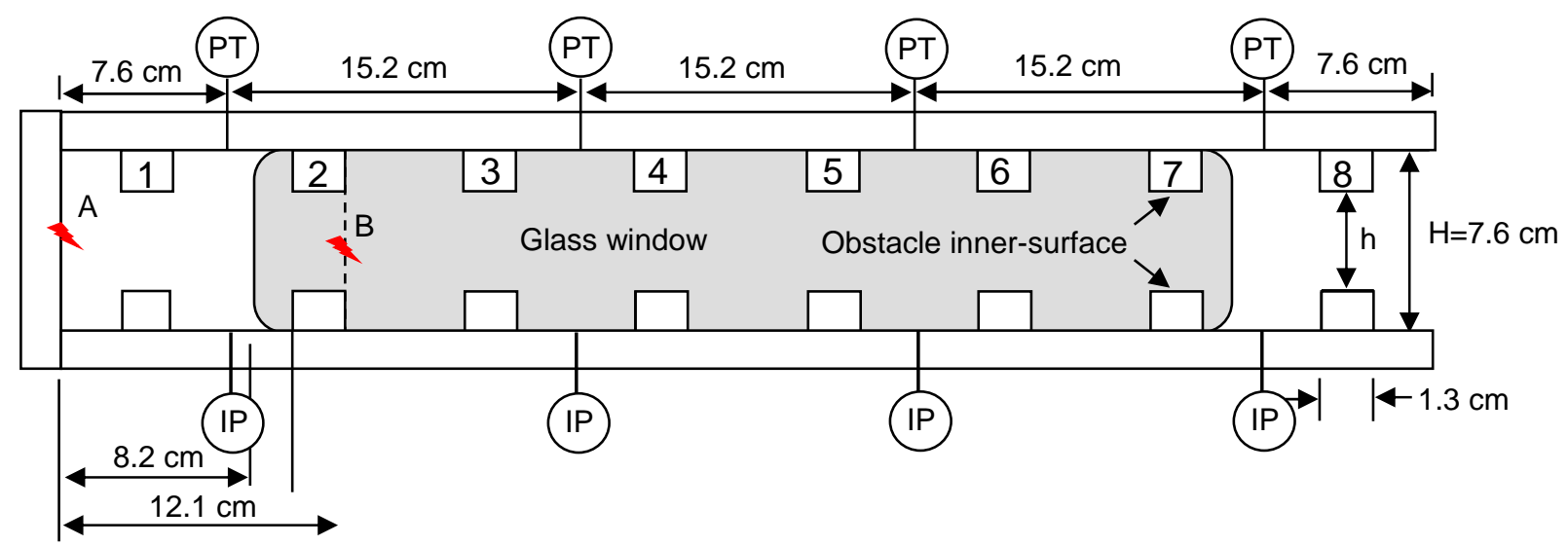

Figure 2: Schematic of optical module showing instrument port locations. For ignition site B the first two obstacles are removed and a plug is inserted reaching up to the dashed line. 
As shown in Fig. 3, visualization of the flame is obtained by positioning the optical module perpendicular to the parallel light produced by a single-pass schlieren system. The schlieren system consists of a 35 Watt Xenon arc lamp, two $25.4 \mathrm{~cm}$ diameter parabolic mirrors, and a Photron 1024 PCI high speed digital camera. In most of the tests the camera was operated with a $1.5 \mu$ s shutter and at 3000 frames per second with a spatial pixel resolution of $1028 \times 524$. Flame acceleration was enhanced through an array of top and bottom-surface mounted obstacles that were distributed along the entire channel length at an equal spacing corresponding to one channel height. The $1.3 \mathrm{~cm}$ thick obstacles spanned the width of the channel producing a twodimensional geometry.

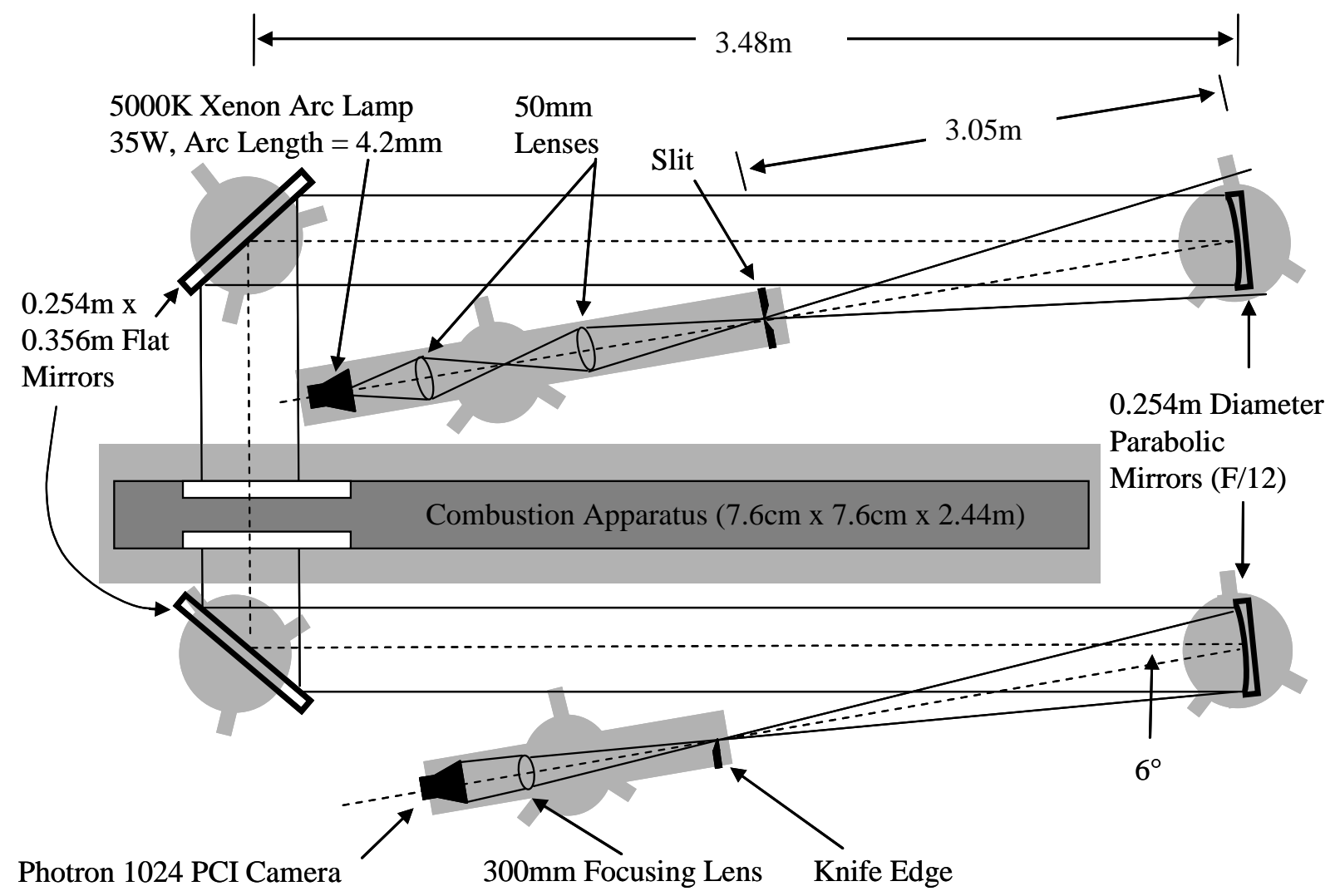

Figure 3: Schlieren photography setup

All the experiments were carried out using stoichiometric methane-air, which was prepared in a separate mixing chamber via the method of partial pressures. The mixing chamber was equipped with an impeller that was driven externally by a pneumatic motor. After fifteen minutes of mixing and thirty minutes of evacuation of the channel down to a pressure of $0.2 \mathrm{kPa}$ absolute, the methane-air mixture was loaded into the apparatus to a final pressure of $47 \mathrm{kPa}$ absolute. Ignition of the mixture is facilitated through the capacitive discharge of approximately $250 \mathrm{~mJ}$ of energy through an automotive spark plug mounted on the center of one of the end flanges, which is designated as position A in Fig. 2. In some limited testing the end wall was extended to the position of obstacle 2 so that the igniter is in the field-of-view, designated as position B in Fig. 2. 


\section{Effect of BR on flame acceleration}

The effect of the obstacle BR was investigated by varying the distance between the obstacle's inner-surfaces for three different cases $(\mathrm{BR}=1-\mathrm{h} / \mathrm{H}=0.33,0.5$, and 0.67). Fig. 4 shows the pressure transients recorded at eight axial positions along the entire channel length that is filled with obstacles with a BR of 0.33. Also shown in Fig. 4 are the flame time-of-arrivals, denoted by triangle symbols, measured at the same axial locations as the pressure traces by ionization probes mounted on the opposite wall. The average flame velocity deduced from adjacent ionization probes is indicated between the pressure traces. There is no noticeable pressure rise ahead of the flame during flame propagation in the first half of the channel, where an average flame velocity of $237 \mathrm{~m} / \mathrm{s}$ is achieved at roughly $1 \mathrm{~m}$ from the ignition end wall. Compression waves start to develop ahead of the flame just after mid-span of the channel where the flame velocity is above the speed of sound in the unburned gas. The compression waves coalesce to form a shock wave by the last pressure transducer that is located $15 \mathrm{~cm}$ from the end wall. The flame at that point follows closely behind the shock wave. The maximum flame velocity achieved at the end of the channel is $708 \mathrm{~m} / \mathrm{s}$. Since the speed of sound in the products is roughly $1000 \mathrm{~m} / \mathrm{s}$, it is possible that if the channel was longer flame acceleration would continue. The shock wave reflects off the end wall and propagates back through the combustion products towards the ignition end. Note that transition to detonation is not observed in the length of the channel or at the end of the channel following shock reflection.

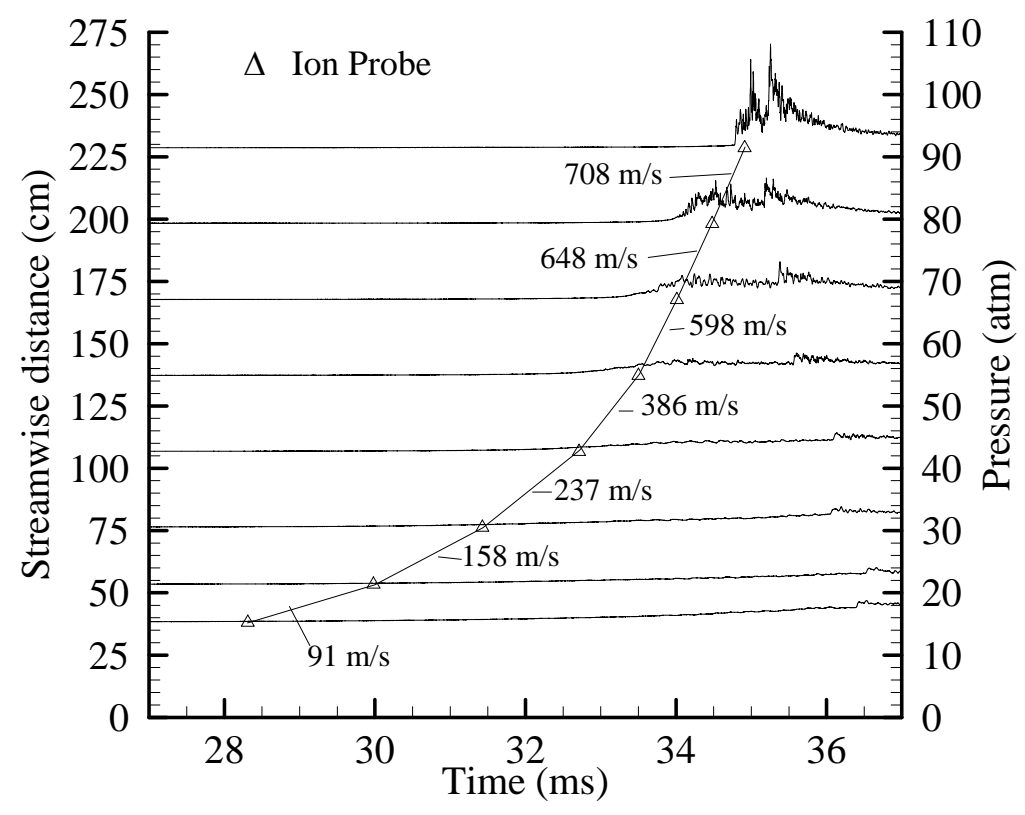

Figure 4: Pressure traces and flame time-of-arrival corresponding to sensors positioned down the entire length of the channel. Average flame velocities shown are based on time-of-arrival. $(\mathrm{BR}=0.33$, ignition position $\mathrm{A})$

The flame velocity measured in the channel equipped with three different blockage ratio obstacles, i.e., 0.33, 0.5, 0.67, is provided in Fig. 5. Each curve represents an average based on at least five tests performed at each blockage ratio. The error bars represent the standard deviation in the velocity data measured in the five tests. The initial flame acceleration 
immediately following ignition is most pronounced in tests where the channel is filled with the largest BR obstacles. For example, at a distance of $0.7 \mathrm{~m}$ the flame velocity is highest in the 0.67 BR obstacles and lowest in the 0.33 BR obstacles. Beyond this point the flame velocities corresponding to $\mathrm{BR}=0.5$ eventually become the highest near the end of the channel. The flame velocity for the $0.67 \mathrm{BR}$ obstacles levels out near the end wall at a lower velocity compared to the other two lower BR obstacles. For all three BR obstacles there is an inflection in the flame velocity versus distance curve that occurs at a flame velocity value just above the speed of sound in the unburned gas, i.e., $400 \mathrm{~m} / \mathrm{s}$. This is the speed where compressibility effects are expected to become important.

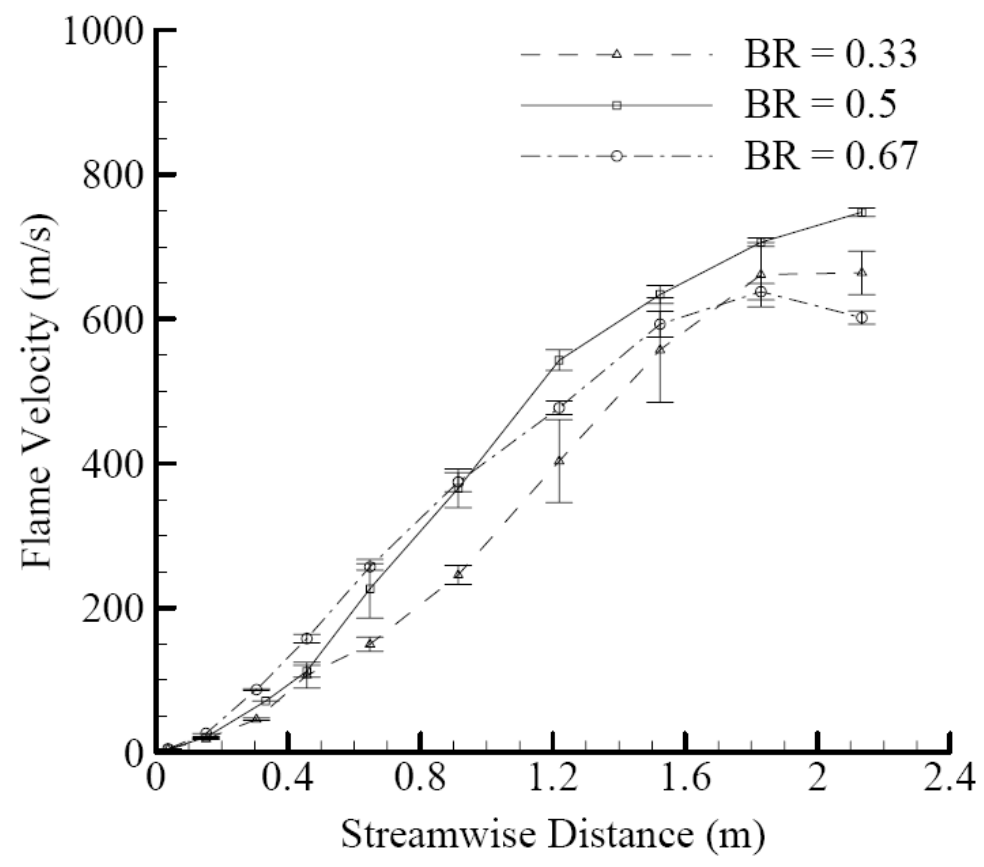

Figure 5: Effect of BR on flame acceleration down the entire length of the channel (ignition position A)

This general trend of the influence of obstacle BR on flame acceleration has also been observed in tubes equipped with orifice plates [5]. Ciccarelli et al. [5] pointed out that for larger BR orifice plates $(\mathrm{BR}>0.43)$ flame acceleration is strongly influenced by the recirculation zone that develops between the plates as well as the shear layer that separates the core flow with the bounded recirculation zone. Visualization of the unburned gas flow field ahead of the flame allows for a more complete picture of influence of these two flow structures on the flame acceleration process.

\section{Flame acceleration visualization}

Visualization of the flame is obtained using the schlieren system shown schematically in Fig. 3. The size of each schlieren image is restricted to a height of $7.6 \mathrm{~cm}$ and a length of 25.4 $\mathrm{cm}$, corresponding to the channel side cut-out height and the diameter of the parabolic mirror, respectively. Figure 6 shows six synchronized top $(x-z)$ and side $(x-y)$ view schlieren photographs of the flame during the early stages of flame acceleration. 

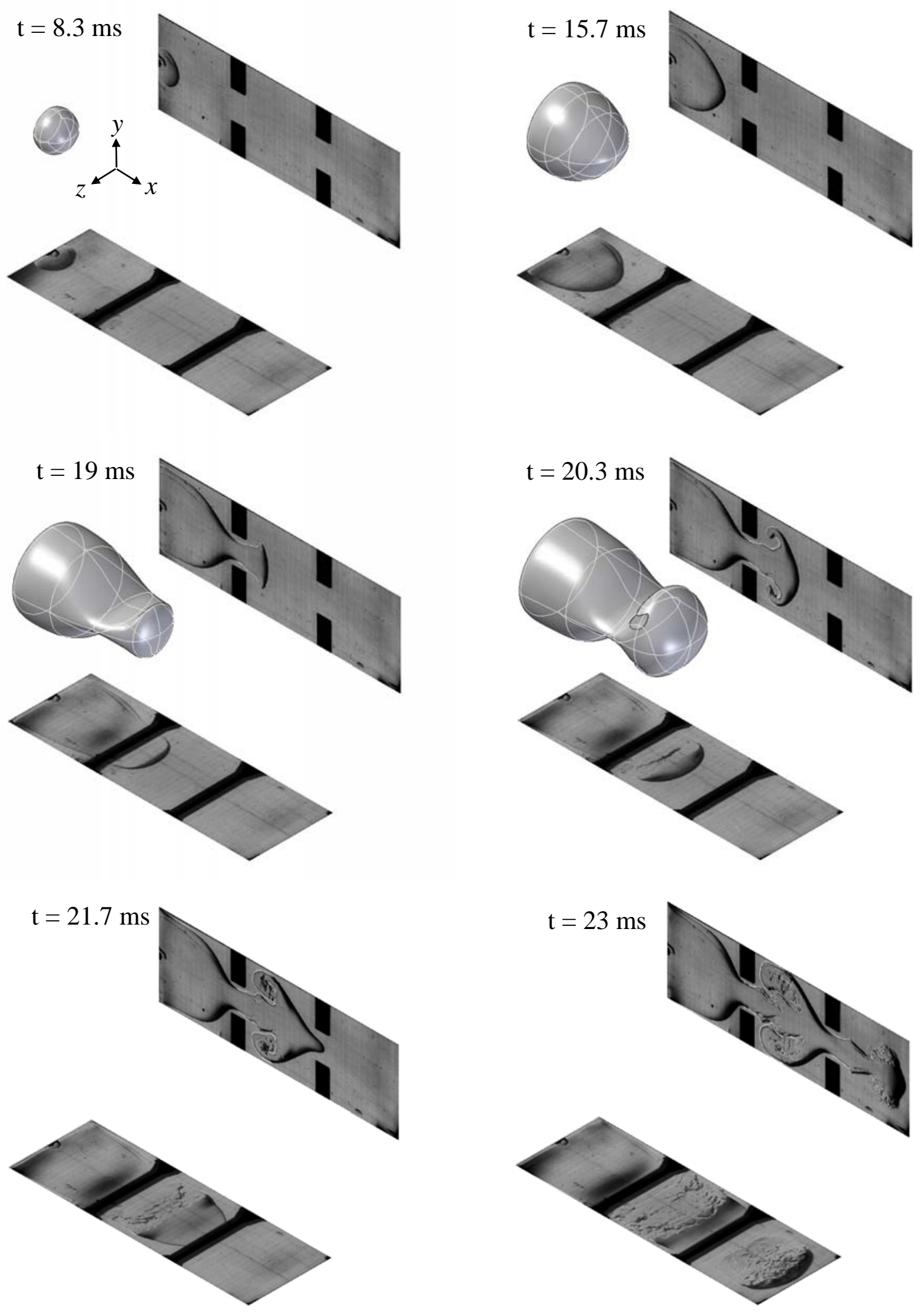

Figure 6: Three dimensional flame shapes constructed from schlieren images. Field of view includes $0.67 \mathrm{BR}$ obstacles 2 through 4 . 
The field of view extends from just after obstacle 2 to just before obstacle 5 . The obstacle locations and numbering scheme that will be used here on in is shown in Fig. 2. For these experiments, the ignition source was placed within the field of view by removing obstacles 1 and 2, and extending the end wall forward by $12 \mathrm{~cm}$ to the position of the downstream face of obstacle 2, i.e., ignition position B in Fig. 2. The side and top-view images were obtained in separate tests by placing the obstacles on the top/bottom walls and the sidewalls, respectively. The side-view schlieren images were obtained with the channel in the position shown in Fig. 1. Since there is no optical access through the top and bottom surfaces of the channel, the top-view schlieren images were obtained by re-orientating the obstacles and securing them directly to the window surface. The synchronization of the schlieren images was possible since negligible differences in centerline flame position with time were observed for the two separate tests.

The commercial CAD software package, Solid Edge V.17 [10], was used to create the three-dimensional revolved protrusion using the flame outline from the top and side-views, respectively. The information required to complete the protrusion was the assumption of an elliptical cross-sectional flame shape that intersects both schlieren outlines in the top and sideviews. The three-dimensional rendered flame shape was produced only for the first four times where a continuous smooth flame surface could be clearly seen. Figure 7 shows the centerline flame velocity measured from the photographs along with the flame area determined from the 3D CAD model. The vertical dashed lines in the graph indicate the times corresponding to the schlieren images in Fig. 6.

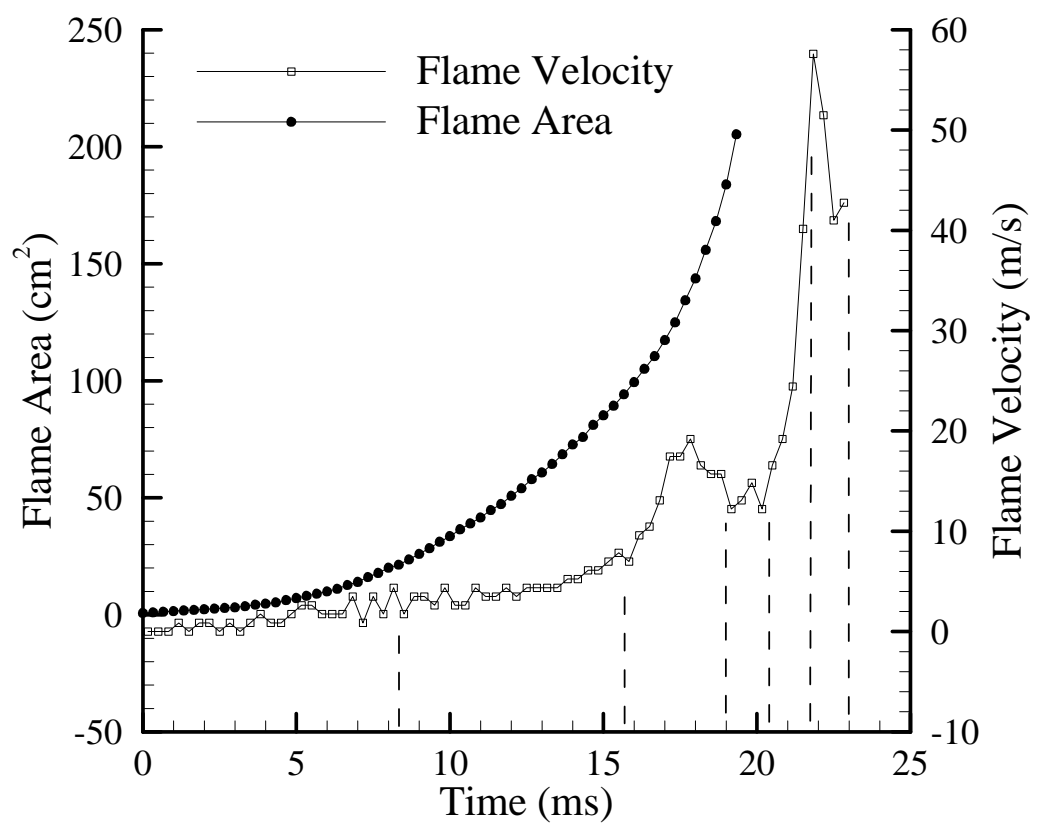

Figure 7: Flame area and velocity time history inferred from schlieren video $(\mathrm{BR}=0.67)$. Dotted vertical lines correspond to the times for the video frames shown in Fig. 6.

Combustion is initiated in the spark plug gap and a flame propagates radially forming a spherical shape until the flame reaches the end wall behind the spark plug, see the image pair at $8.3 \mathrm{~ms}$ in Fig. 6. This initial propagation appears to be symmetrical as the schlieren images 
indicate similar outlines in both the top and side-views. During this early period the flame area experiences a monotonic growth that is roughly proportional to the square of the flame ball diameter. This increase in the flame area leads to a similar growth in the volumetric burning rate which in turn results, on average, in a monotonic increase in the flame velocity relative to a fixed observer. From Fig. 7 it is clear that the centerline flame velocity experiences well defined oscillations superimposed on a monotonic increase. The oscillations observed in the flame velocity are due to non-uniformities in the flow field of the unburned gas into which the flame propagates, and not an increase in the volumetric burning rate due to a sudden increase in the flame area. For example, as the flame ball size approaches the width of the channel, lateral flame movement slows and the unburned gas is forced to turn downstream. This, along with a natural contraction of the unburned gas flow as it approaches the first obstacle in the field of view, causes the flame to stretch in the $x$-direction. This can be seen in the side-view image at $15.7 \mathrm{~ms}$ in Fig. 6. The flame tip is convected downstream by the high speed contracted unburned gas flow. This manifests itself as a sudden surge in the flame velocity observed between $15.7 \mathrm{~ms}$ and $17 \mathrm{~ms}$ in Fig. 7.

Immediately after the surge in flame velocity the unburned gas flow expands downstream of the obstacle causing the flame velocity to decrease. The schlieren photograph taken at $19 \mathrm{~ms}$ is representative of this flow expansion period. In the top-view, the flame remains laminar and takes on a parabolic profile corresponding to the unburned gas velocity profile across the channel height. As the flame approaches each channel surface $(\mathrm{t}>19 \mathrm{~ms})$, the assumption of an elliptical cross-section flame area is no longer valid. The flame shape becomes roughly planar with distortions occurring mainly in the $x-y$ plane. After passing through the first obstacle the edges of the flame appear to be entrained into a vortex pair positioned downstream of the obstacle, see the $x-y$ plane photograph at $20.3 \mathrm{~ms}$ in Fig. 6 . The combustion appears to be laminar over most of the flame surface except at the core of the vortex roll up where the surface is slightly wrinkled. The wrinkling is also apparent in the top-view photograph at $20.3 \mathrm{~ms}$. The entrainment of the flame results in an exponential increase in the flame area between $15.7 \mathrm{~ms}$ and $20.3 \mathrm{~ms}$. Interestingly, the flame velocity drops during this roll-up period, indicating that the increase in the flame area and therefore volumetric burning rate is not sufficient to counteract the unburned gas flow expansion just ahead of the flame tip.

As the flame tip approaches the next obstacle in the field of view it accelerates due to the contraction of the unburned gas flow. This acceleration produces a thin flame tongue that passes through the second obstacle, see the $x-y$ plane photograph at $21.7 \mathrm{~ms}$ in Fig. 6 . This corresponds to a second surge in the centerline flame velocity between $20.3 \mathrm{~ms}$ and $21.7 \mathrm{~ms}$ in Fig. 7 . Both schlieren photograph views at $21.7 \mathrm{~ms}$ indicate that the vortex core becomes turbulent resulting in a highly wrinkled flame surface. This complicated flame shape prohibits flame area measurements using the $\mathrm{CAD}$ modeling technique due to the difficulty of accurately resolving the flame surface.

The side-view photograph taken at $23 \mathrm{~ms}$ indicates that as the flame propagates through the second obstacle it is not rolled into the vortex pair, as was the case in the first obstacle. The flame tip takes on a mushroom like shape just before the third obstacle located at the end of the field of view. The downstream surface of the mushroom shaped flame tip remains laminar but the upstream surface appears highly turbulent in both views shown at $23 \mathrm{~ms}$. The flame velocity drops significantly between $22 \mathrm{~ms}$ and $23 \mathrm{~ms}$ as the flame approaches the third obstacle and the flame tip "mushrooms". 
The oscillations in the centerline flame velocity discussed above do not appear in the flame velocity data shown in Fig. 5 because of the large distance between the ionization probes. The centerline flame velocity inferred from the schlieren video in the first channel module (0.08 $\mathrm{m}<\mathrm{x}<0.53 \mathrm{~m}$ ) for the three BR obstacles is provided in Fig. 8. It should be noted that the velocity data in Fig. 8 was obtained using ignition site $A$, which is different from the data in Fig. 7 that was obtained using ignition site B. As was observed in the ionization probe data in Fig. 5 the average flame acceleration is more significant the higher the BR. The oscillations in the flame velocity are very apparent in the video based velocity measurements shown in Fig. 8 for all three BR obstacles. There is a clear trend in the data that the flame tip velocity accelerates as it propagates through the obstacle and then decelerates between the obstacles. As discussed above this is due to the contraction and expansion of the unburned gas flow that convects the flame tip. The amplitude of the oscillations is more significant in the higher BR obstacles because of the more severe flow contraction.

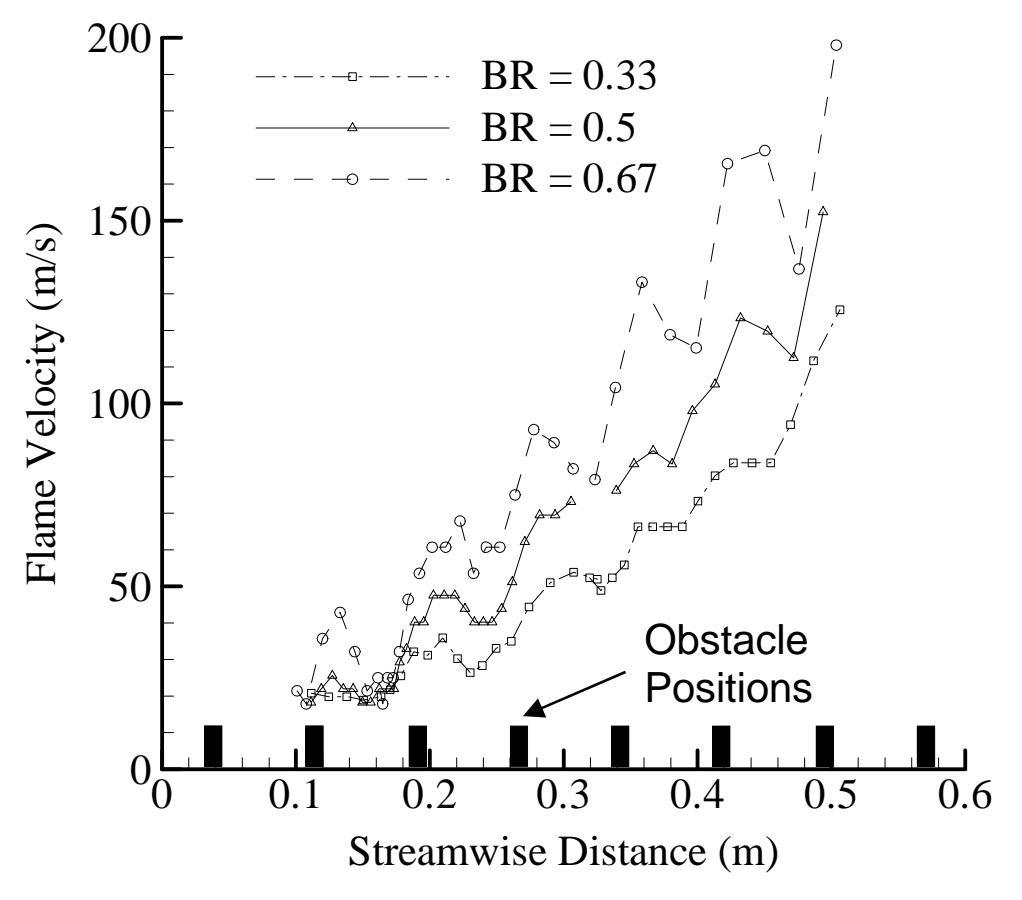

Figure 8: Flame velocity deduced from the schlieren video for different BR obstacles (ignition site A)

\section{Unburned gas flow visualization}

In order to better understand the phenomenon driving the evolution of the flame shape and flame tip centerline velocity it is imperative to obtain information on the unburned gas flow immediately ahead of the flame. In order to accomplish this, a novel visualization technique was developed where a small amount of helium, that is significantly less dense than the fuel-air mixture, is injected in the unburned gas to act as a tracer in the schlieren photographs. The helium is injected into the channel one second before ignition through a $6.4 \mathrm{~mm}$ hole in the top surface of the channel. The helium enters the channel with a mean jet velocity of $3.7 \mathrm{~m} / \mathrm{s}$. 

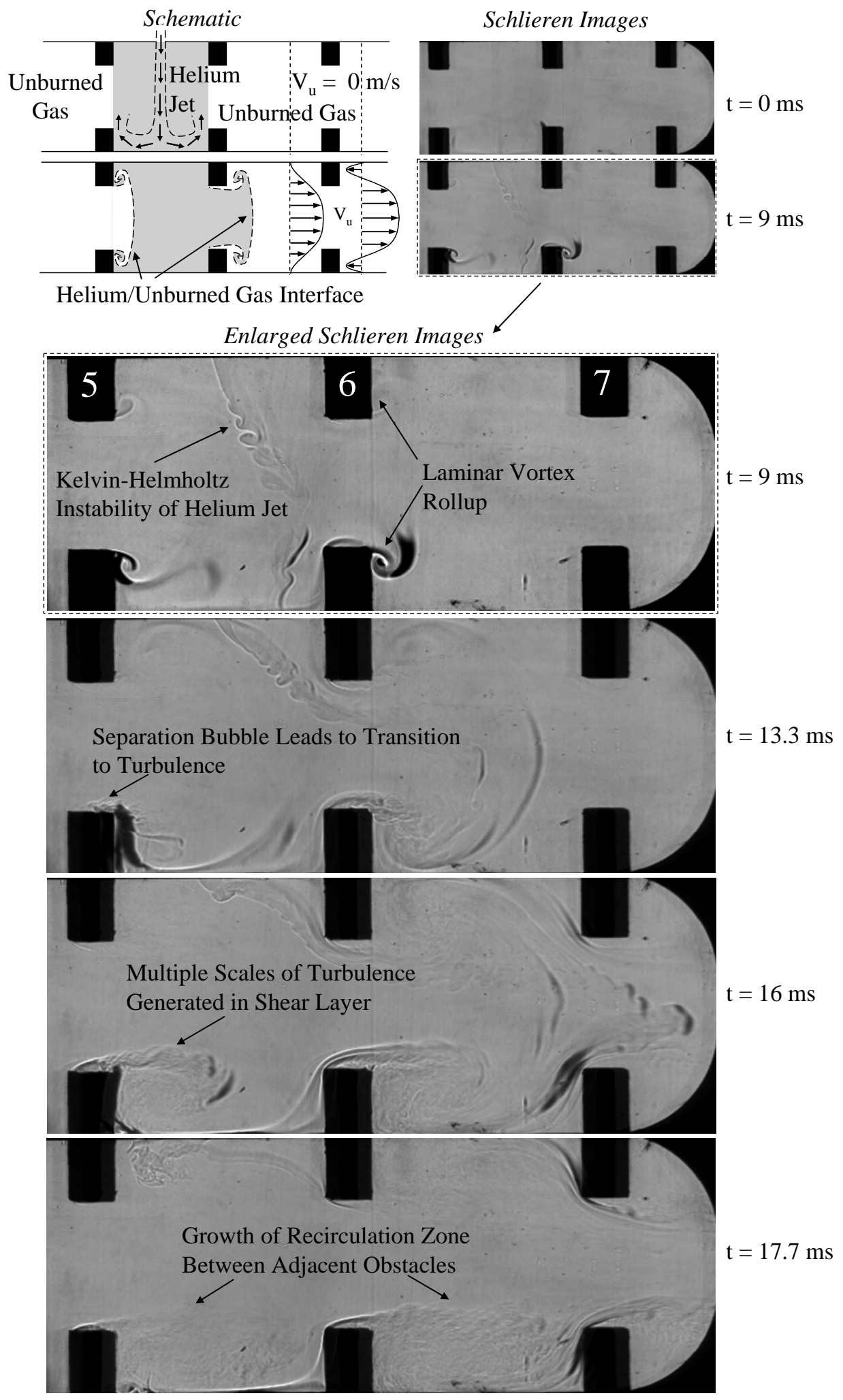

Figure 9: Schlieren photographs and schematic of the development of the unburned gas flow field ahead of the flame using helium gas as a tracer. Field of view includes 0.5 BR obstacles 5 through 7. 
As shown schematically in Fig. 9, the helium enters the channel as a jet and then mixes with the fuel-air mixture in a region bounded by adjacent obstacles. The schlieren photographs shown in Fig. 9 are from a test using ignition position A where the helium is injected midway between obstacles 5 and 6.

After ignition, the expansion of the flame ball, not seen in Fig. 9, induces an unburned gas flow to the right. Since the flame area continually increases with time, see Fig. 7, the unburned gas mass flow rate through the channel core also increases with time. In the schlieren photograph taken at $9 \mathrm{~ms}$ after ignition the helium jet is convected to the right and roughly follows the parabolic axial velocity profile across the channel. A combination of RayleighTaylor and Kelvin Helmholtz instabilities leads to the perturbations observed on the upstream jet boundary. The schlieren photographs show the evolution of the interfaces separating the fuel-air mixture and the helium-diluted fuel-air mixture at obstacles 5 and 6.

The unburned gas flow separates at the obstacle inner-surface to form a pair of vortices downstream of each obstacle. The generation of the vortex pair seen in the schlieren photograph at $9 \mathrm{~ms}$ in Fig. 9 is similar to the axisymmetric vortex produced by ejecting fluid from a circular nozzle via an impulsively-started piston [11]. Geometrically the observed flow phenomenon is more representative of the rolling-up process associated with wing tip vorticies. The thin boundary layer at the inner-surface of the obstacle separates at the downstream edge as a planar shear layer rolls up into a spiral entraining irrotational fluid in the process. The initial vortex roll up is laminar with a well defined spiral and core.

As the unburned gas channel core flow velocity increases ahead of the flame, the flow at the upstream edge of the obstacle detaches and forms a new, unstable shear layer that disrupts the roll up of the initial laminar vortex. For example, in the photograph taken at $13.3 \mathrm{~ms}$ the laminar vortex that formed at obstacle 5 is displaced away from the channel centerline. The flow separation forms a "separation bubble" that is clearly visible next to the inner-surface of obstacle 5 at $16 \mathrm{~ms}$. The separation bubble is visible due to the backflow of helium from downstream of obstacle 5. The unstable shear layer then rolls up itself, entraining the laminar vortex in the process. The new vortex that forms is fully turbulent with no discernible spiral structure and the shear layer has some large-scale eddy structure imbedded. With time the unstable shear layer grows in length extending towards the downstream obstacle inner-surface and the vortex grows in size as more fluid is entrained. Once the vortex reaches the channel wall it lengthens until it fills the gap between obstacles forming a turbulent "recirculation zone," see the photograph at $17.7 \mathrm{~ms}$ in Fig. 9. The recirculation zone is separated from the laminar channel core flow by a strong turbulent shear layer that stretches from the edge of one obstacle to roughly the edge of the adjacent obstacle.

\section{Simultaneous visualization of the flame and the unburned gas}

The visualization of the unburned gas flow field provides insight into the evolution of the flame shape, combustion mode, and flame centerline velocity down the length of the channel. Since the unburned gas flow develops with time, the flame front, especially the tip, will experience different unburned gas flow conditions along the channel length. In Figs. 10-12 schlieren photographs show the simultaneous progression of the flame and the development of the unburned gas flow field ahead of the flame for tests corresponding to the three respective obstacle BRs. 


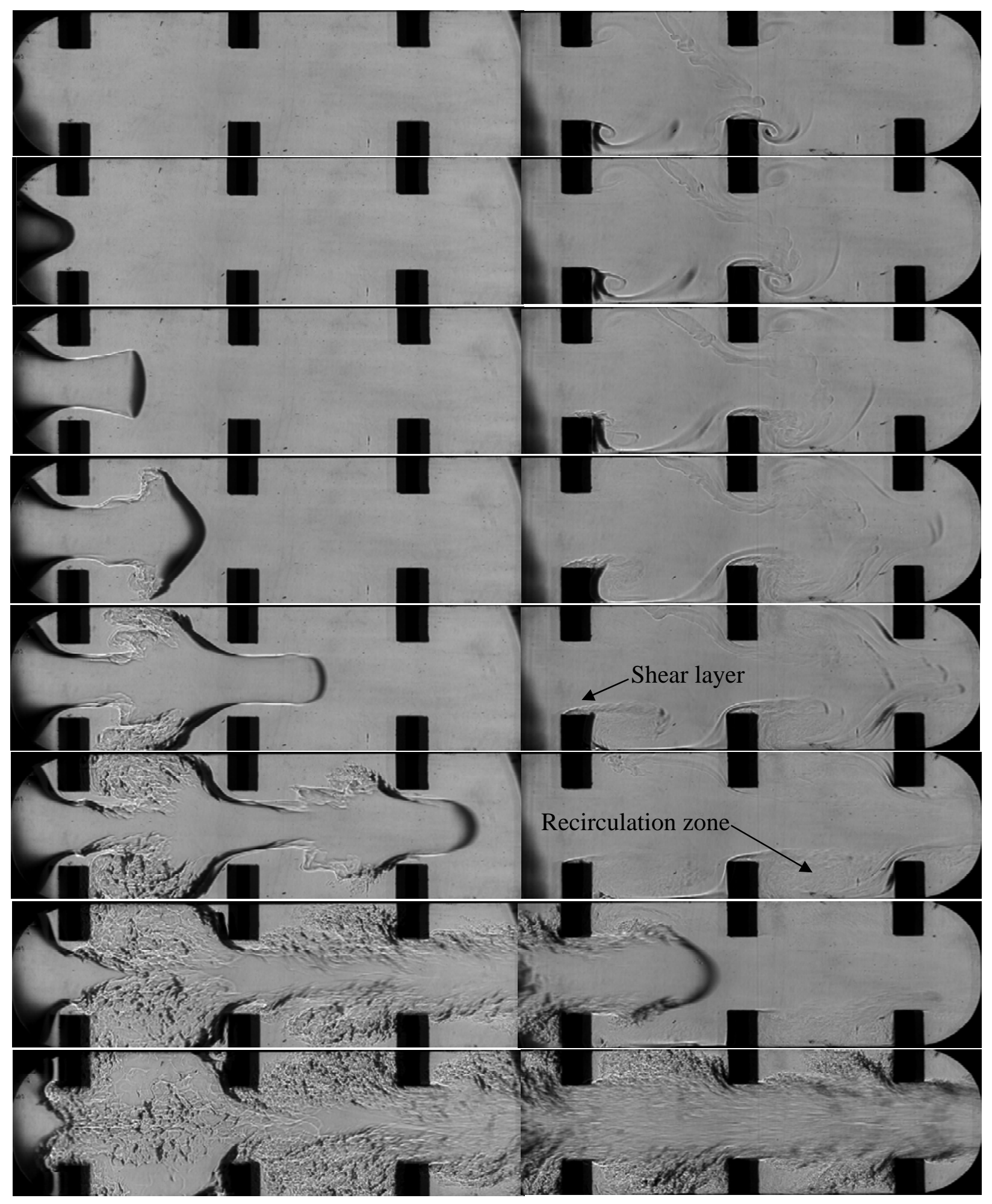

Figure 10: Schlieren video showing the simultaneous development of the flame surface and the unburned gas flow field ahead. Field of view includes 0.5 BR obstacles 2 through 7. Inter-frame time is $1 \mathrm{~ms}$. 

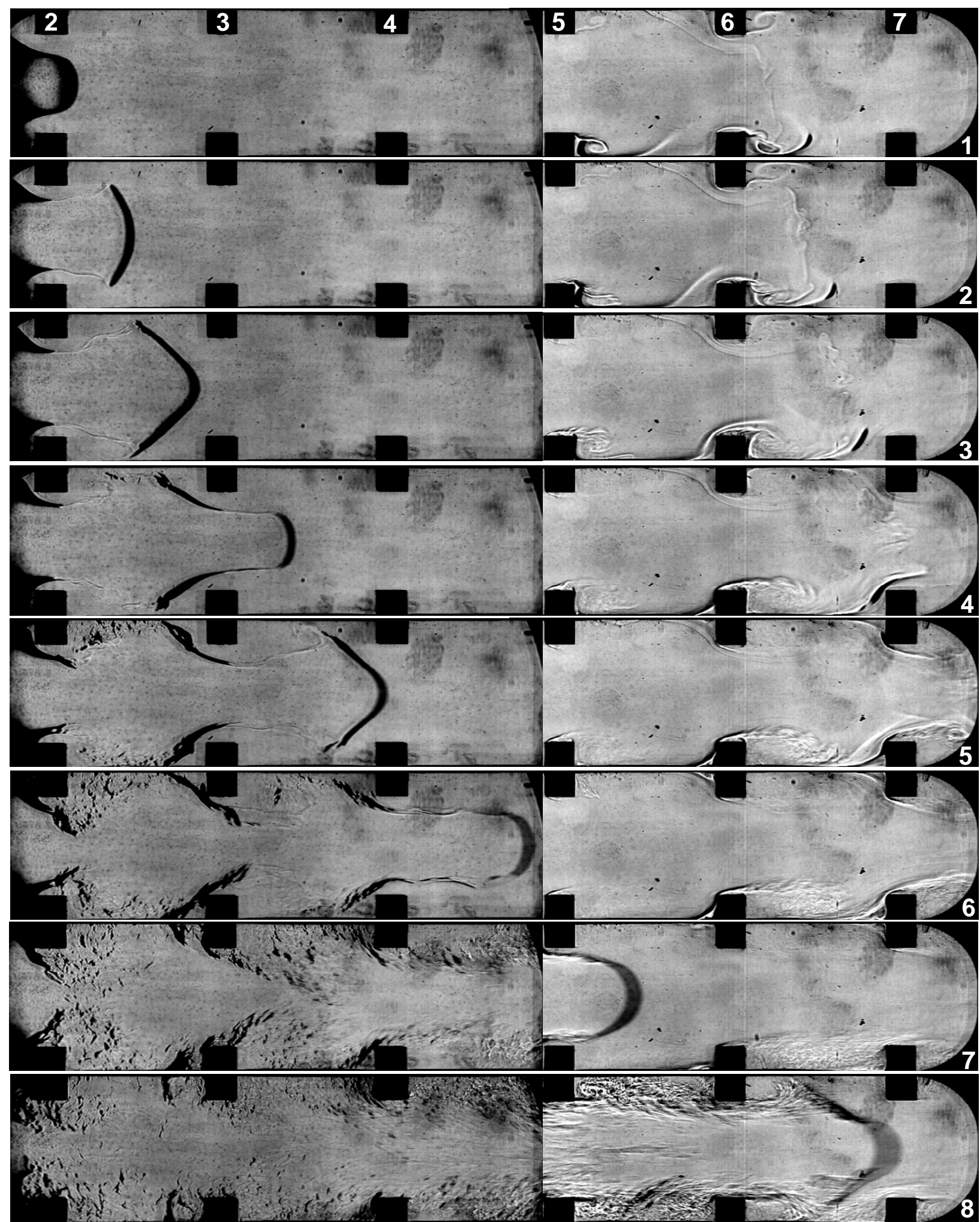

Figure 11: Schlieren video showing the simultaneous development of the flame surface and the unburned gas flow field ahead. Field of view includes 0.33 BR obstacles 2 through 7. Inter-frame time is $1.33 \mathrm{~ms}$. 

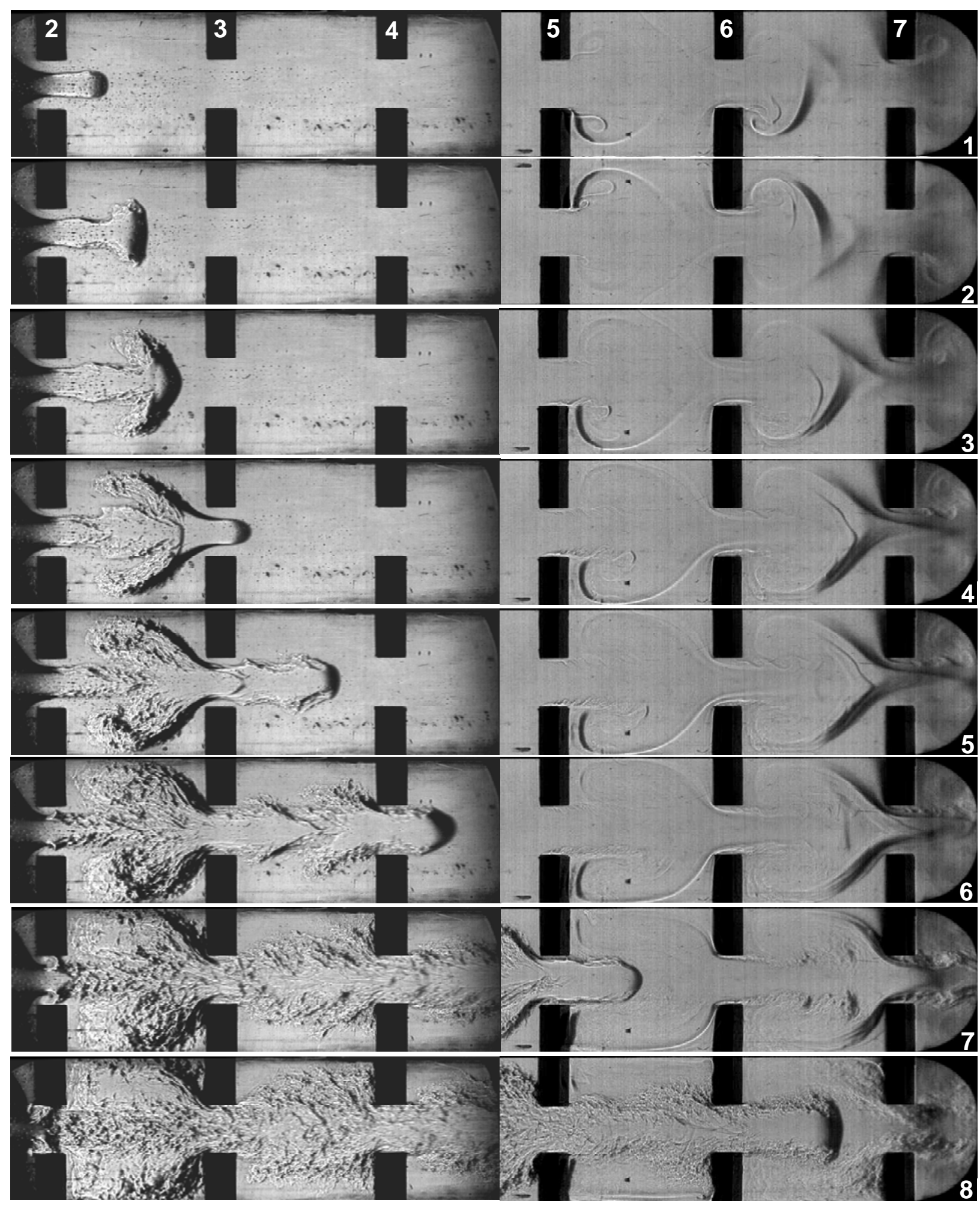

Figure 12: Schlieren video showing the simultaneous development of the flame surface and the unburned gas flow field ahead. Field of view includes 0.67 BR obstacles 2 through 7 . Inter-frame time is $0.67 \mathrm{~ms}$. 
These schlieren images are a composite of two separate but synchronized videos that were positioned side by side to create a total field of view of $7.6 \mathrm{~cm} \mathrm{x} 44.5 \mathrm{~cm}$, which corresponds to the dimensions of the entire side cut-out in the optical module. For these tests ignition position A was used and therefore the first obstacle that appears in the field of view is actually obstacle 2, see Fig. 2. The video frame and obstacle numbers are provided in Fig. 10 for reference. Recall on average the flame tip acceleration is stronger for the higher BR obstacles. For example, based on the data provided in Fig. 8 the centerline flame tip velocity measured at roughly $0.5 \mathrm{~m}$ from the igniter end wall, for the 0.67, 0.5, and $0.33 \mathrm{BR}$ obstacles is $200 \mathrm{~m} / \mathrm{s}, 150 \mathrm{~m} / \mathrm{s}$ and $125 \mathrm{~m} / \mathrm{s}$, respectively. The camera is operated at 3000 frames per second which yields a frame every $0.333 \mathrm{~ms}$. The time between frames in Figs. 10-12 is chosen such that the flame enters and exits the field of view over the same number of frames. This results in an inter-frame time of $0.67 \mathrm{~ms}$, $1 \mathrm{~ms}, 1.33 \mathrm{~ms}$ for the $0.67,0.5$, and $0.33 \mathrm{BR}$ obstacles, respectively. Helium is injected before ignition between obstacles 5 and 6 . It should be noted that the unburned gas flow field immediately ahead of the flame in any frame is roughly the same as the flow field highlighted by the helium that is injected between obstacles 5 and 6. Fluid flow, and consequently vortex roll up, is initiated at each obstacle following the passage of the sound wave produced at the onset of flame growth. This results in a slight delay in the initial development of vortices downstream of obstacles further from the ignition point.

\section{Channel with 0.5 BR orifice plates}

A general description of the flame acceleration phenomenon for the intermediate size 0.5 BR obstacles shown in Fig. 10 is discussed in this section. The unburned gas flow field between obstacles 5 and 7 shown in Fig. 9 is the same as that shown in Fig. 10. After the flame passes obstacle 2 (first obstacle in the field-of-view), it propagates around the vortex outer boundary, burning to the channel wall. As seen in the fourth frame in Fig. 10, this produces a "mushroom" shape flame tip. This behavior is different from that observed at the earlier times where the channel core flow velocity is lower and the flame is entrained into the vortex, see the photograph at $20.3 \mathrm{~ms}$ in Fig. 6. By the third frame the unburned gas flow has separated from the upstream edge of the obstacle (visualized at obstacles 5 and 6) and the newly formed turbulent shear layer effectively deflects the flame from the initial laminar vortex. By the fifth frame the turbulent shear layer extends to roughly half the distance between the obstacles. The shear layer prevents the flame from being entrained into the vortex downstream of obstacle 2. The flame eventually burns back towards obstacle 2 through the turbulent vortex at a speed on the order of $10 \mathrm{~m} / \mathrm{s}$. The flame does not propagate immediately to the upstream face of obstacle 2; it appears that the flow in the corner region remains laminar. Unburned gas from the upstream corner feeds the shear layer downstream of obstacle 2. By the seventh frame the mixture in the shear layer between obstacles 2 and 3 is consumed by the turbulent flame propagating in the vortex. The flame then propagates upstream around obstacle 2 into the upstream corner region, see the eighth frame.

After the first few obstacles flame propagation in the streamwise direction is dominant resulting in a long and narrow flame shape. By the sixth frame the shear layer extends across to the next obstacle and a turbulent recirculation zone is established. At this point in time the flame tip is still laminar and propagates at a velocity of $70 \mathrm{~m} / \mathrm{s}$ relative to a fixed observer. The shear layer extending from the upstream edge of obstacles 3 deflects the flame from the obstacle innersurface around the separation bubble. The flame burns into the recirculation zone between obstacles 3 and 4 just before reaching obstacle 4 where the shear layer would be expected to be 
weakest. The turbulent flame then burns back towards obstacle 3 . In the seventh frame the flame reaches the upstream face of obstacle 4. The flame surface at that location appears very rough indicating that turbulent conditions prevail in the corner region resulting in a high burning velocity. Once the flame penetrates the flow near the wall it is quickly convected into the shear layer downstream of obstacle 4. As a result, the flame is able to burn into the separation bubble adjacent to the inner-surface of the obstacle. The flame burns into the recirculation zone near obstacle 5 and then burns back to obstacle 4. This phenomenon repeats itself for all the subsequent obstacles. Note for the seventh and eighth frame the flame beyond the fourth obstacle propagates into a helium diluted mixture. In tests performed without helium the flame shape and propagation phenomenon observed are similar.

By the eighth frame the flame extends over all six obstacles and the recirculation zones between obstacles are at various degrees of consumption. Interestingly, the portion of the flame approaching the upstream face of obstacle 2 remains laminar over the duration of the eight frames and thus burns very slowly. Just before the flame leaves the field-of-view, see frame 7, it appears that the turbulent shear layer has not spread to the centerline of the channel and the flame tip is laminar. The flame tip is blurry due to the very fast flame speed and insufficiently fast camera shutter speed. It appears that before compressibility effects set in, the core flow remains laminar due to the short time available for the shear layers to spread towards the channel centerline. Therefore, the flame tip is essentially convected downstream at an ever increasing speed as a result of the burning in the trailing recirculation zones. Communication between the burning recirculation zones and the flame tip occurs via acoustic waves propagating in the combustion products. As the flame accelerates down the channel the average unburned gas velocity increases which results in a higher average circulation and turbulence intensity level at each subsequent recirculation zone encountered by the flame. Both of these contribute to a higher recirculation zone consumption rate. For example, the recirculation zone between obstacles 5 and 6 is mostly consumed between frames 7 and 8, whereas the recirculation zone between obstacles 4 and 5 requires more than two frames for complete consumption.

\section{Channel with 0.33 BR orifice plates}

Schlieren video taken of the flame acceleration in the smaller $0.33 \mathrm{BR}$ obstacles is shown in Fig. 11. The flame tip that enters the first obstacle is broader than that observed in the $0.5 \mathrm{BR}$ obstacles. This is because of the larger core flow area and less flow contraction through the smaller 0.33 BR obstacles. Because of the short obstacle height the vortices that develop downstream of the obstacles quickly reach the channel top and bottom wall. By the second frame a separation bubble develops at the obstacle 2 inner-surface and the flame propagates around the vortex and reaches the channel wall, similar to that observed with the channel equipped with the $0.5 \mathrm{BR}$ obstacles. The flame then burns back into the turbulent vortex and then up to the separation bubble by the eighth frame.

By the fifth frame the shear layer and the vortex extends roughly half way between the obstacles. In the fifth frame it can clearly be seen that the part of the top and bottom edge of the flame between obstacles 3 and 4 follows the shear layer. The flame surface entering the recirculation is largely smooth compared to that observed in the $0.5 \mathrm{BR}$ test, see the sixth frame in Fig. 10, where the flame surface at the same location is more turbulent. As the flame tip advances to the last obstacle and the shear layer becomes more turbulent the flame is able to burn into the recirculation zone earlier, see the eighth frame. The biggest difference between the 0.33 $\mathrm{BR}$ and $0.5 \mathrm{BR}$ obstacles is the flame surface distribution. In the smaller BR case the 
recirculation zones burn out quicker due to the shorter obstacle height. For example, by the time the flame reaches the end of the field of view in frame 8 most of the recirculation zones have been consumed. This is in contrast to the $0.5 \mathrm{BR}$ obstacle case, see frame 8 in Fig 10, where the flame has advanced further in the channel yet there remains significantly more unburned mixture in the recirculation zones behind the flame tip. Similar to the $0.5 \mathrm{BR}$ obstacle case the flame tip remains smooth as it approaches the final obstacle indicating that the flow in the channel core remains laminar.

\section{Channel with 0.67 BR orifice plates}

Schlieren video taken of the flame acceleration in the 0.67 BR obstacles is shown in Fig. 12. As the flame propagates through the first obstacle in the field of view it is severely stretched due to the large flow contraction through obstacle 2. The flow contraction is made worse by the separated flow at the obstacle so the flame tip is effectively only half the height of the obstacle opening. The vortex and recirculation zones develop downstream of the obstacles and the flame propagates in a manner similar to that observed in the 0.5 and $0.33 \mathrm{BR}$ obstacles. The shear layer that develops downstream of the obstacles appear to have large-scale structures not observed in tests with the smaller BR obstacles. For example, in the seventh frame the shear layer that develops downstream of obstacle 6 has two large eddies. It is possible that these large flow structures develop due to the interaction of the two shear layers that are in close proximity to each other for the $0.67 \mathrm{BR}$ obstacles. It is also evident that combustion at the flame tip is more turbulent compared to that observed with the lower BR obstacles. In the last frame it appears that the flow through the last obstacle in the field of view is fully turbulent as the shear layers merge.

\section{Flame propagation Mechanism}

For the two-dimensional obstacles tested with BR in the range of 0.33 and 0.67 , the early development of the unburned gas flow field and flame shape appears to be universal. A comparison of the last frame in Figs. 10-12 indicates that the general distribution of the flame area appears to be the same for all three BR obstacles. However, the rate at which the flame area evolves is very different as evident by the different inter-frame time for each figure. Therefore, the rate of flame area development and the corresponding flame tip acceleration is dependent on the obstacle BR.

The development of the unburned gas flow field ahead of the flame dictates the evolution of the flame surface. Except for very early times, the flame surface consists of a laminar flame tip propagating downstream in the channel core and the rest of the flame surface propagates transversely into the recirculation zones between obstacles. As the turbulent flame burns into the recirculation zones the combustion products expand into the channel core behind the flame tip. This is clearly evident in frame 8 of Fig. 10 where streaks can be observed in the combustion products in the core, close to the flame tip. The streaks occur due to an insufficiently fast shutter speed to "freeze" the motion of the density gradient field that exists in the core. Note the streaks do not appear in the $0.67 \mathrm{BR}$ obstacle image because a faster shutter speed was used. The expansion of the combustion products produced over the lateral surface of the flame is responsible for the unburned gas flow in the channel core. Theoretically, for a planar flame propagating in an obstacle free duct, the combustion products are stationary for flame velocities below the speed of sound of the combustion products [4]. 
a) $\mathrm{BR}=0.33$

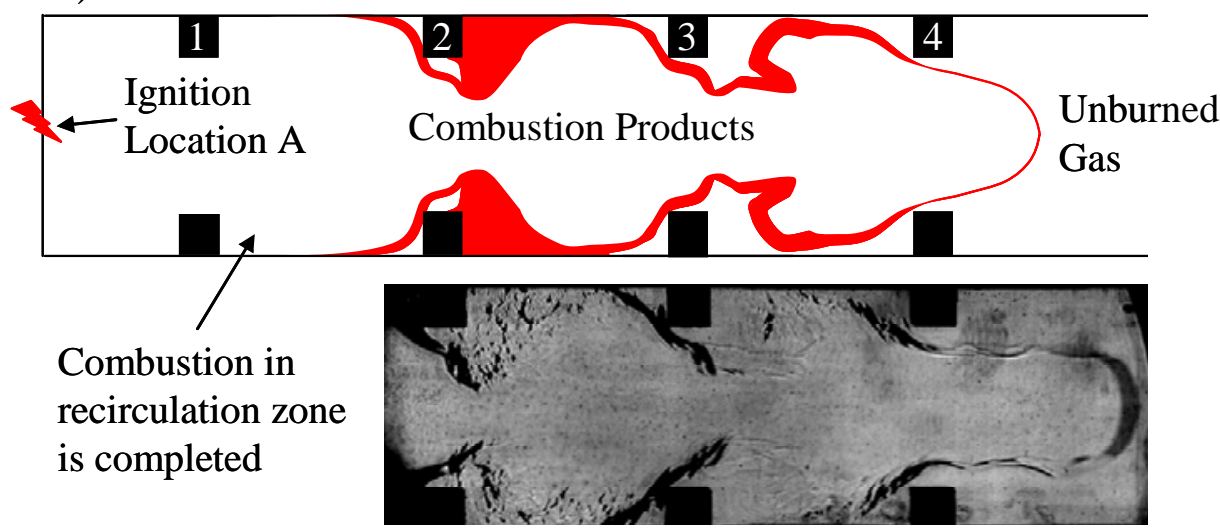

b) $\mathrm{BR}=0.5$

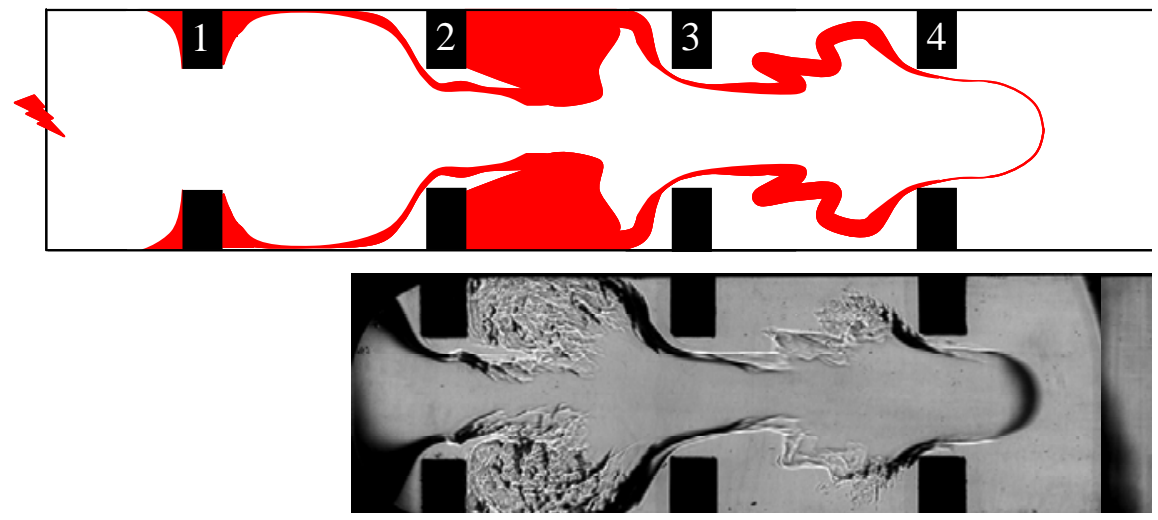

c) $\mathrm{BR}=0.67$

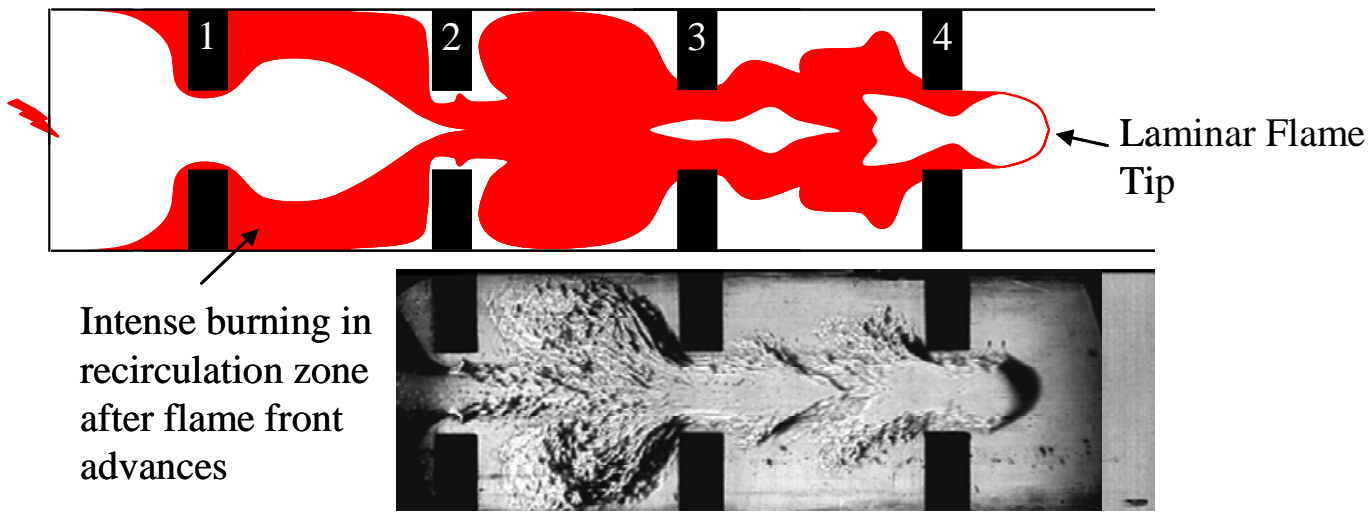

Figure 13: Schematic of the effect of obstacle BR on total flame area. Field of view includes ignition to obstacle 4 with flame tip located between obstacles 4 and 5 for each BR. 
In an obstacle laden duct, the flame surface extends to the ignition end, such that parts of the flame surface are transverse to the axial flame propagation direction. Expansion of the gas across the transverse portions of the flame will generate a flow in the combustion products at the channel core, as discussed above. This flow convects the flame tip and unburned gas forward. Combustion along the lateral surface behind the flame front persists for longer times for higher BR obstacles. This is illustrated in Fig. 13, which shows the flame shape when the tip reaches obstacle 4 for the three different blockage ratios. Note that turbulence and three-dimensional effects make it difficult to identify the exact flame shape from the schlieren images. The larger flame surface associated with the higher blockage ratio obstacles results in a larger volumetric burning rate. Since combustion at the flame tip remains laminar over the observation time, the observed flame tip velocity is essentially equal to the unburned gas velocity immediately ahead of the flame. The combination of a smaller core area and larger volumetric burning rate results in a higher centerline flame velocity for the larger BR obstacles.

\section{Conclusions}

The characterization of the flow field ahead of a flame front in an obstacle-laden channel was investigated for three blockage ratios for the early stages of flame acceleration. The compilation of a sequence of 3D rendered flame shapes constructed from schlieren images show that the flame tip is smooth and distortions are mainly restricted to the $x$ - $y$ plane. A helium tracer visualization technique revealed the development of recirculation zones downstream of each obstacle. The development of the recirculation zones governs the combustion in the gap between obstacles. Oscillations in centerline flame velocity are due to the acceleration and deceleration of the unburned gas as it flows through each obstacle. It was found that initial rates of flame acceleration were higher for large blockage ratios due to enhanced turbulence production and increased bulk burning rate associated with larger flame areas. Future work includes the investigation of the flow field ahead of the flame front at the later stages of flame acceleration, where compressibility effects such as shock/flame interactions dominate. In addition, a largeeddy simulation of the development of the unburned gas flow field will be carried out by setting the ignition end boundary to a transient inflow condition based on the measured flame area timehistory. Quantitative data describing the development of the recirculation zones will be obtained from the numerical simulations to complement the experimental observations.

\section{Acknowledgements}

This work was supported by the Natural Sciences and Engineering Research Council of Canada.

\section{References}

1. Chapman, W. R., and Wheeler, R. N. V., Journal Chem. Soc. London, pp. 2139-2147, 1926.

2. Lee, J. H. S., and Moen, I. O., Progress in Energy and Combustion Science, 6:359-389, 1980.

3. Oran, E.S. and Gamezo, V.N., Origins of DDT in gas phase combustion, Combustion and Flame, 148:4-47, 2007.

4. Ciccarelli, G. and Dorofeev, S., Flame acceleration and transition to detonation in ducts, Progress in Energy and Combustion Science, 34: 499-550, 2008.

5. Ciccarelli, G., Fowler, and C., Bardon, M., Shock Waves, 14: 161-166, 2005.

6. Veser, A., Breitung, W. and Dorofeev, S., $4^{\text {th }}$ International Symposium on Hazards, Prevention and Mitigation of Industrial Explosions, France, 2002. 
7. Adams, G.K. and Pack D.C., Some observations on the problem of transition between deflagration and detonation, Proceedings of the Combust Institute, 7:812-819, 1959.

8. Kuznetsov, M.S., Matsukov, I.D., Alekseev, V.I., Dorofeev, S.B. $17^{\text {th }}$ International Colloquium on the Dynamics of Explosions and Reactive Systems, Heidelberg, Germany, 1999.

9. Lee, J. H. S., Kynstautas, R., and Chan, C.K., Proceedings of the Combustion Institute, Vol. 20: 1663-1672, 1985.

10. Solid Edge V.17, UGS Corp, http://www.ugs.com/en_us/

11. Didden, N., J. Appl. Math. Phys., 30: 101-116, 1979. 